\title{
Unravelling the controversies surrounding host immune response to helminth and malaria parasites
}

\author{
Dennis Adu-Gyasi ${ }^{*}$, Margaret T Frempong ${ }^{2}$, Harry Danwonno ${ }^{1}$, Alberta Bedford Moses ${ }^{3}$ and Ben Gyan ${ }^{4}$ \\ ${ }^{1}$ Kintampo Health Research Centre, P O Box 200, Kintampo, Brong Ahafo, Ghana \\ ${ }^{2}$ Kwame Nkrumah University of Science and Technology (KNUST), Department of Molecular Medicine, PMB, Kumasi, Ghana \\ ${ }^{3}$ Cape Coast Teaching Hospital, Cape Coast, Ghana \\ ${ }^{4}$ Noguchi Memorial Institute for Medical Research, Legon Accra, Ghana
}

\section{Introduction}

Humans are hosts to nearly 300 species of parasitic worms; which have co-evolved over hundred million years, with over 70 species of protozoa including malaria parasites [1,2]. Commonly, helminth and malaria cause great impact in terms of mortality, morbidity and contributions to the Quality/Disability Adjusted Life Years (QALYs/ DALYs) [3] of their hosts. Since communication in the immunological labyrinth involves cells, cytokines and antibodies together with host natural defense mechanisms, it is important to understand the mechanisms that might be involved in hosts response to helminths and malaria coinfections [4], a knowledge paramount in the control of these infections. How much of influence does the environment have on the level of immune response to an infection needs to be unraveled? Why do I say this?

\section{Humoral and Cell mediated Immunity in Infection}

Apart from the innate immune response involved in fighting against all infections, the adaptive immune response which comprises antibodies (humoral) and cell mediation (T cells) is vital to successfully stop infection from being established [5]. When T-cell Receptors complexes with MHC I or II with peptide of processed antigens on an Antigen Presenting Cell (APC), coupled with CD3 activation, the cells proliferate and differentiate targeting the antigen [1,5-8] for a successful clearance. The outcome of the effector cells produced from such activations are dependent on the cytokine milieu of the host, and this environment is largely influenced by the phenotypes of the genes of the T-cell regulatory factors. The complex life cycles of helminth and malaria parasites in their hosts obviously affect the T-cell proliferation and differentiation pathway. One important question that remains unanswered is whether one can just categorize the two infections into the Th1 and Th2 arms as have been tried previously?

\section{Helminth immunology}

Hookworms survive relatively well in an immunologically hostile environment, although the Th2 phenotype is associated with partial protection. The parasite may promote its survival by secreting a molecular screen of immune- suppressive agents and, possibly, by stimulating the appearance of regulatory T-cell populations $[9,10]$. Hookworm has the capacity to downregulate the FceR1 on basophils and mast cells which in turn explains the protective effect in hookworm infection against environmental allergens and responses that lessens the detrimental effects in autoimmunity $[9,11,12]$. Fortunately, Pritchard et al, 2007 showed that from a parasitological standpoint, potentially protective FceRI-dependent immune responses are not blocked in hookworm infection but rather mediated by secreted parasite immune suppressants and the induction of regulatory leukocyte populations [13].

In a Schistosome-endemic area, egg-positive people had significantly higher levels of specific antibodies and IFN- $\gamma$ in contrast to egg negative individuals that had significantly higher circulating IL4, IL-13 and IL-21 [14]. Previous studies suggesting that anti-helminth immune responses fall into a Th1 (pro-inflammatory) and Th2 (antiinflammatory) dichotomy with resistance to infection being associated with Th2 responses failed to fully explain resistance, susceptibility to infection and re-infection in people resident in helminth endemic areas. For example, both Th1 and Th2 responsiveness appear compromised in schistosomiasis patients, and within the Th2 compartment, IL-5 responses were suppressed while IL-4 production was relatively intact [15]. There is no clear pattern between Th1 or Th2 cytokine responses and infection intensity. The existence of a regulatory subset of T-cells (Treg), which modulate the effects of Th1 and Th2 responses through the immunosuppressive cytokines interleukin-10 (IL-10) and transforming growth factor beta (TGF $\beta$ ) have been characterized [1618]. The balance between Th1, Th2 and Treg responses determine the outcome of helminth infections $[14,16-18]$ a process largely attributed to the plastic nature of T-cells.

\section{Plasmodium species immunology}

Malaria pathogenesis is complex and this mostly involves immunologic and non-immunologic mechanisms. In malaria parasite immunology, humoral immune response is elicited to the extracellular stage and cell mediated immune (CMI) response; involving largely T-cells, to the intracellular phase of the parasites' development [5] with the involvement of some peripheral blood cells and lymphocyte subsets [8]. It has been alluded that CD4+ T-cell responses are associated with controlling the intracellular phase of malaria parasites' infection [5]. But since the parasite biology and kinetics at the various stages of the life cycle differ significantly, one would expect the immune response elicited to the parasite stages to be dissimilar and even if CD4+ plays a

${ }^{\star}$ Correspondence to: Dennis Adu-Gyasi, Kintampo Health Research Centre, P O Box 200, Kintampo, Brong Ahafo, Ghana, E-mail: dennis.adu-gyasi@ kintampo-hrc.org

Received: July 15, 2019; Accepted: August 08, 2019; Published: August 12, 2019 
significantly role, it might be in a different subset bearing in mind the concept of CD4+ T-cell plasticity $[5,16,18]$. Little has been documented of the very role $\mathrm{CD} 4+\mathrm{T}$-cells play in the immune response to the pre-erythrocytic stage of malaria parasite infection. But because of records of antibodies in vaccination $[6,19]$, which indicate response to antigens of the extracellular stage, such as circumsporozoite protein (CSP) used for RTS,S malaria vaccine and liver-stage antigen 1 (LSA1), the involvement of CD4+ cells in receiving antigens from APCs and activating B-cells cannot be discounted [6,20]. Another form of T-cells, $T C R-\gamma \delta$ cells which make up $1 \%-10 \%$ of circulating lymphocytes in human exhibit marked increase in human Plasmodium spp parasite infections, which could remain elevated for more than a month even after treatment $[8,20]$. $T C R-\gamma \delta$ play important cytotoxic role in malaria parasite infection [20], operate through cytolytic and proinflammatory molecules, their increase correspond to an increase in IFN- $\gamma$ produced by T-bet+ Th1 cells, Natural Killer (NK) cells, natural regulatory cells (Tregs; to suppress immune reaction), natural killer T (NKT) cells and CD8+ T-cells $[6,20]$.

Basically, Th1/Th2 paradigm has been proposed to fight against malaria infection in human and this has not been enough to explain the immune response put forward in infected people. Follicular CD4+ $\mathrm{T}$ (Tfh) cells producing IL-21 cytokines have been reported in immune adults living in endemic areas of $P f$. Though activation of Th17 subset of CD4+ cells producing IL-17A cytokine occurs, there have not been any identified and defined role during Plasmodium infections $[5,16,18]$. The regulatory T-cells are extremely important to control the inflammatory process in malaria $[5,6,16,21]$. Immunoglobulin-E (IgE), which is usually known to be under the influence of IL-4 from Th2 cells, has been seen as the preserve of helminth infection [22]. But total and parasite-specific IgE levels have been reported in cases of malaria which supports the fact that IFN- $\gamma$ also cause upregulation of low affinity IgE receptor because of IgE elevation [9] and high levels of IgE correlate with protection against severe malaria [9].

\section{Immunology in co-infections of Helminth and Plasmo- dium species}

Helminth trigger Th2 cell differentiation producing IL-4, IL-5, IL13, IL-9 and IL-25 to obtain effector cells that will induce IgE antibodies from B-cells and further suppress regulator genes of the Th1 arm of the immune response. At the larval phase of early helminth infection, Th1 cells are induced and their fecundity trigger the Th2 usual response [1]. The immune response elicited is dependent on the infection stage just as in the case of malaria parasite infection $[5,23]$, which though it is Th1, activates the Th2 arm at the pre-erythrocytic stage [6,9]. The life cycle of the infecting pathogens together with the cytokine environment determines the response one mounts to helminth, malaria parasites or their co-infections [20]. The questions of how the coexistence of helminths and Plasmodium parasites influence the immunological responses to each species need answers [20,23].

\section{Conclusions}

The interplay of the various cells after receiving antigens from APCs mainly the CD4+, CD8+, their activated forms and the TCR$\gamma \delta$ (has also an APC independent pathway of activation) with their regulatory role through the cytokines and molecules they produce is worth studying in helminth, malaria parasite or their co-infections in an endemic place to unravel the controversies surrounding immune response elicited by their host in more structured studies considering environmental influence.

\section{Acknowledgements}

Great appreciation goes to Kintampo Health Research Centre for the support to carry out the project to facilitate the review. Also, thank you to Portia Amoah for her layman's review and comments to the essay.

\section{Authors contributions}

Concept and review carried out by DAG, HD, AB, MTF and BG. Draft essay was written by DAG, reviewed and accepted by all authors.

\section{Declaration}

The authors have declared that no competing interests exist.

\section{References}

1. Ridley JW (2012) Parasitology for Medical and Clinical Laboratory Professionals DELMAR CENGAGE Learning, USA p. 336.

2. Zaiss M, Rapin A, Lebon L, Dubey L, Mosconi I, Sarter K, et al. (2015) The Intestina Microbiota Contributes to the Ability of Helminths to Modulate Allergic Inflammation. Immunity 43: 998-1010.

3. Hotez PJ, Diemert D, Bacon KM, Beaumier C, Bethony JM, et al. (2013) The Human Hookworm Vaccine. Vaccine 31 Suppl 2: B227-232. [Crossref]

4. Allen JE, Maizels RM (2011) Diversity and dialogue in immunity to helminths. Nat Rev Immunol 11: 375-388. [Crossref]

5. Luckheeram R, Zhou R, Verma A, Xia B (2012) CD4+T Cells: Differentiation and Functions. Clin Dev Immunol 2012: 925135.

6. Abbas A, Lichtman A, Pillai S (2014) Basic immunology: functions and disorders of the immune system. ( $4^{\text {th }}$ Edn) Elsevier Saunders, USA.

7. Actor J (2014) Introductory Immunology: Basic Concepts for Interdisciplinary Applications. Academic Press, UK.

8. Kenneth K, Marshall A, Josef T, Marcel L, Oliver W, et al. (2016) Williams Hematology ( $9^{\text {th }}$ Edn) McGraw-Hill Education, New York.

9. Conrad DH (1990) Fc epsilon RII/CD23: the low affinity receptor for IgE. Annu Rev Immunol 8: 623-645. [Crossref]

10. Hewitson J, Grainger J, Maizels R (2009) Helminth immunoregulation: The role of parasite secreted proteins in modulating host immunity. Mol Biochem Parasitol 167 $1-11$.

11. Adkinson F, Stephen H, Bruce B, Robert F, Wesley B, et al. (2014) Middleton's Allergy 2-Volume Set 8th Edition. Allergy principles and practise, Elsevier Saunders, USA.

12. Smallwood T, Giacomin P, Loukas A, Mulvenna J, Clark R, et al. (2017) Helminth Immunomodulation in Autoimmune Disease. Frontiers in Immunology 8: 453.

13. Pritchard D, Hooi D, Brown A, Bockarie M, Caddick R, et al. (2007) Basophil competence during hookworm (Necator americanus) infection. The American journal of tropical medicine and hygiene 77: 860-865.

14. Milner T, Reilly L, Nausch N, Midzi N, Mduluza T, Maizels R, et al. (2010) Circulating cytokine levels and antibody responses to human Schistosoma haematobium: IL- 5 and IL- 10 levels depend upon age and infection status. Parasite Immunol 32: 710-721.

15. Grogan JL, Kremsner PG, Deelder AM, Yazdanbakhsh M (1998) Antigen-specific proliferation and interferon-? and interleukin-5 production are down-regulated during Schistosoma haematobium infection. J Infect Dis 177: 1433-1437.

16. Perez-Mazliah D, Langhorne J (2015) CD4T-cell subsets in malaria: TH1/TH2 revisited. Front Immunol 5: 671.

17. McKee AS, Pearce EJ (2004) CD25+ CD4+ cells contribute to Th2 polarization during helminth infection by suppressing Th1 response development. The Journal of Immunology 173: 1224-1231.

18. Nakayamada S, Takahashi H, Kanno Y, O'Shea JJ (2012) Helper T cell diversity and plasticity. Curr Opin Immunol 24: 297-302. [Crossref]

19. Imoukhuede EB, Ventura R, Imbault N, van Schooten H, Leroy O (2010) European Malaria Vaccine Initiative: portfolio and perspectives for the future. Human vaccines 6: 146-150. 
20. Sanou S, Régis W, André L, Amidou D, Alphonse O, et al. (2012) Haematological parameters, natural regulatory $\mathrm{CD} 4+\mathrm{CD} 25+\mathrm{FOXP} 3+\mathrm{T}$ cells and gd $\mathrm{T}$ cells among two sympatric ethnic groups having different susceptibility to malaria in Burkina Faso Guillaume. BMC Research Notes 5: 76.

21. Ateba-Ngoa U, Adegnika A, Zinsou J, Kassa R, Smits H, et al. (2015) Cytokine and chemokine profile of the innate and adaptive immune response of schistosoma haematobium and plasmodium falciparum single and co-infected school-aged children from an endemic area of Lambaréné, Gabon. Malaria journal 14: 94.
22. Nacher M, Gay F, Singhasivanon P, Krudsood S, Treeprasertsuk S, et al. (2000) Ascaris lumbricoides infection is associated with protection from cerebral malaria. Parasite Immunol 22: 107-113.

23. Salazar-Castañon V, Legorreta-Herrera M, Rodriguez-Sosa M(2014) Helminth Parasites Alter Protection against Plasmodium Infection. BioMed research international.

Copyright: (2019 Adu-Gyasi D. This is an open-access article distributed under the terms of the Creative Commons Attribution License, which permits unrestricted use, distribution, and reproduction in any medium, provided the original author and source are credited. 